\title{
Ultrasound guidance in regional anesthesia: state of the art review through challenging clinical scenarios
}

\author{
Brian D Sites' \\ John G Antonakakis ${ }^{2}$ \\ 'Departments of Anesthesiology \\ and Orthopedic Surgery, Dartmouth \\ Medical School, Dartmouth-Hitchcock \\ Medical Center, Lebanon, New \\ Hampshire, USA; ${ }^{2}$ Department \\ of Anesthesiology, University \\ of Virginia Health System, \\ Charlottesville, VA, USA
}

\begin{abstract}
Ultrasound guided regional anesthesia (UGRA) for peripheral nerve blockade is becoming increasingly popular. The advantage of ultrasound technology is that it affords the anesthesiologist the real time ability to visualize neural structures, needle advancement, and local anesthetic spread. Recent data suggest that UGRA generates improved success rates and reductions in performance times in comparison to traditional approaches. Further, the use of ultrasound technology in peripheral nerve blocks has provided insight into needle-nerve interactions, revealing distinct limitations of nerve stimulator techniques. Given that UGRA requires a unique set of skills, formal standards and guidelines are currently being developed by leadership societies in order to foster education and training. This review article, in a case vignette format, highlights important techniques, concepts, and limitations regarding the use of ultrasound to facilitate regional anesthesia. Clinically relevant aspects of ultrasound physics are also discussed.
\end{abstract}

Keywords: ultrasound, regional anesthesia

\section{Introduction}

Regional anesthesia can now compete with propofol/LMA techniques for speed, accuracy, and success. Data are rapidly emerging regarding the improved success rates of ultrasound guided regional anesthesia (UGRA) in comparison to traditional approaches. ${ }^{1-4}$ The following review article, through real case vignettes, will highlight important techniques, concepts, and limitations regarding the use of ultrasound to facilitate regional anesthesia.

\section{Upper extremity Case I (Background)}

You are a 46-year-old anesthesiologist and have been performing nerve stimulator and paresthesia techniques for your entire career. You are interested in learning ultrasound guided regional anesthesia (UGRA) techniques, but are afraid that you will not be able to turn on the ultrasound machine. What background information do you need to know?

Ultrasound is generated when multiple piezoelectric crystals inside a transducer (the probe) rapidly vibrate in response to an alternating electric current. This generates a mechanical form of energy that is simply a "high" frequency sound wave. Ultrasound then travels into the body where upon contact with various tissues, it can be reflected, refracted, and scattered. Structures that reflect ultrasound to a greater degree appear whiter, or more hyperechoic. Structures that reflect ultrasound to a lesser degree are described as hypoechoic and appear darker. Therefore, all structures appear as different shades of gray, allowing for specific tissue diagnosis (eg, needle from nerve). 
State of the art soft-tissue ultrasound is conducted in two dimensions. This means that structures can be imaged either in their short-axis or long-axis (Figure 1). There are two common techniques for needle insertion. These are the in-plane approach and the out-of-plane approach. With the in-plane approach, the needle itself is imaged in its long-axis. This allows the potential for full needle visualization, including the needle tip (Figure 2). With the out-of-plane approach, the needle will be imaged in short-axis, generating a limited view. The downside to the out-of-plane approach is that the operator cannot be assured that the needle tip is being imaged in contrast to part of the shaft. The main downside to the in-plane technique is that the ultrasound beam is very thin and it can be frustrating and difficult to continuously maintain needle imaging. The authors usually favor the in-plane technique for single injection blocks and the out-of-plane technique for continuous blocks and central line placements.

The frequency of the ultrasound system is directly related to the resolution of the system. Resolution generally refers to the ability to image structure detail and to distinguish one object from another. The higher the frequency of the ultrasound, the better the resolution. High end frequencies in clinical UGRA are between 10 and $13 \mathrm{MHz}$. It turns out, however, that higher frequency systems do not penetrate deeply into the body, thus preventing the effective imaging of "deep" structures. This limitation occurs secondary to a process known as ultrasound attenuation. Higher frequency ultrasound attenuates at more superficial depths than lower frequency ultrasound. The clinical reality is that there is a trade off between depth of penetration and resolution. More superficial structures such as the interscalene brachial plexus should be scanned at frequencies greater than $10 \mathrm{MHz}$. For deeper blocks such as lumbar plexus, transgluteal sciatic, and neuraxial procedures, frequencies between 3 and $8 \mathrm{MHZ}$ should allow adequate penetration.

The American Society of Regional Anesthesia and Pain Medicine (ASRA) and the European Society of Regional Anaesthesia and Pain Therapy (ESRA) Joint Committee Recommendations for Education and Training in Ultrasound Guided Regional Anesthesia were recently completed. ${ }^{5}$ This group has recommended 10 important steps to performing an
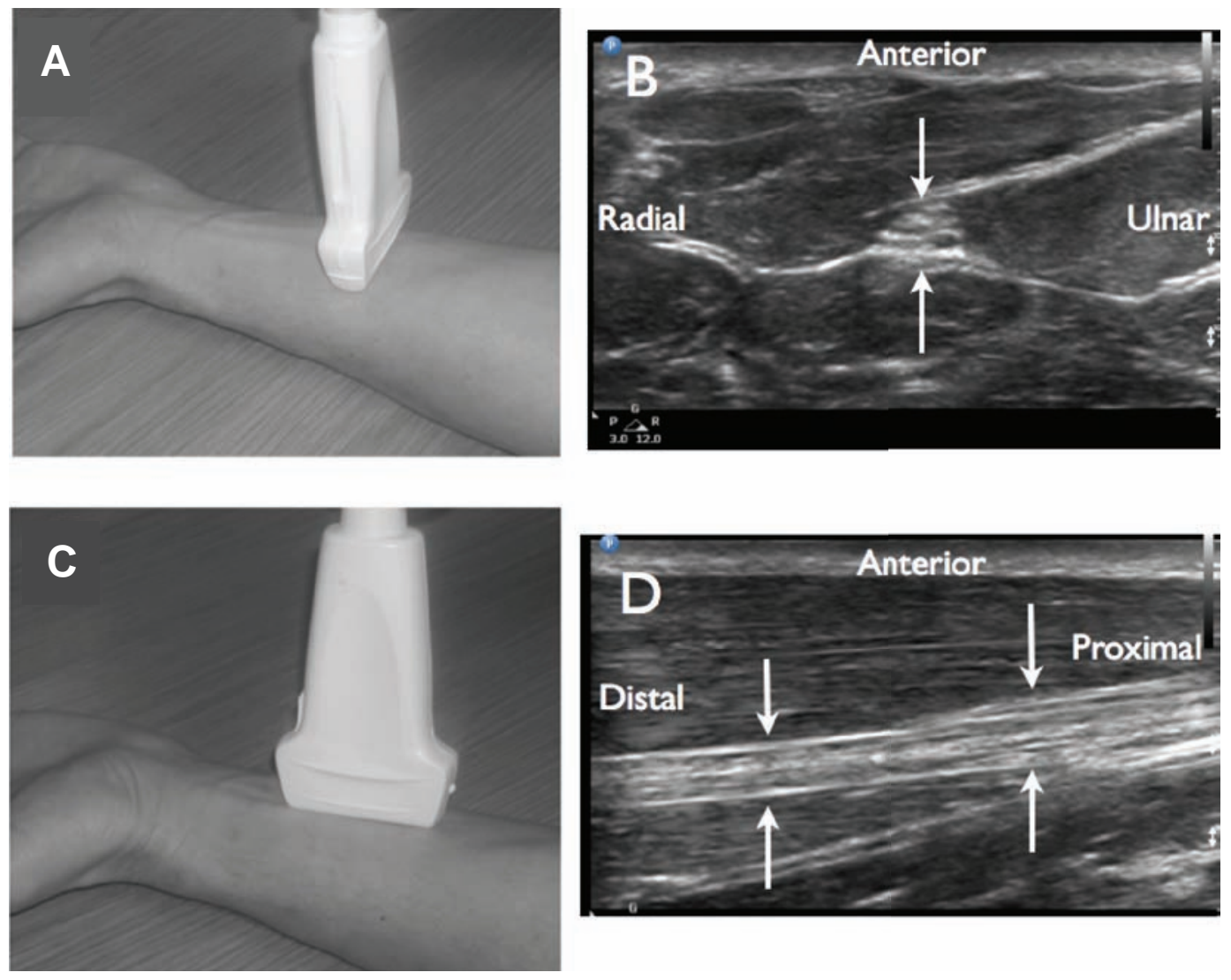

Figure I Two-dimensional ultrasound imaging of neural structures. Transducer placement A). for short axis (cross sectional view) of the median nerve in the forearm B). When the transducer is turned 90 degrees $\mathbf{C}$ ), a long axis view of the median nerve is seen $\mathbf{D})$ ). 


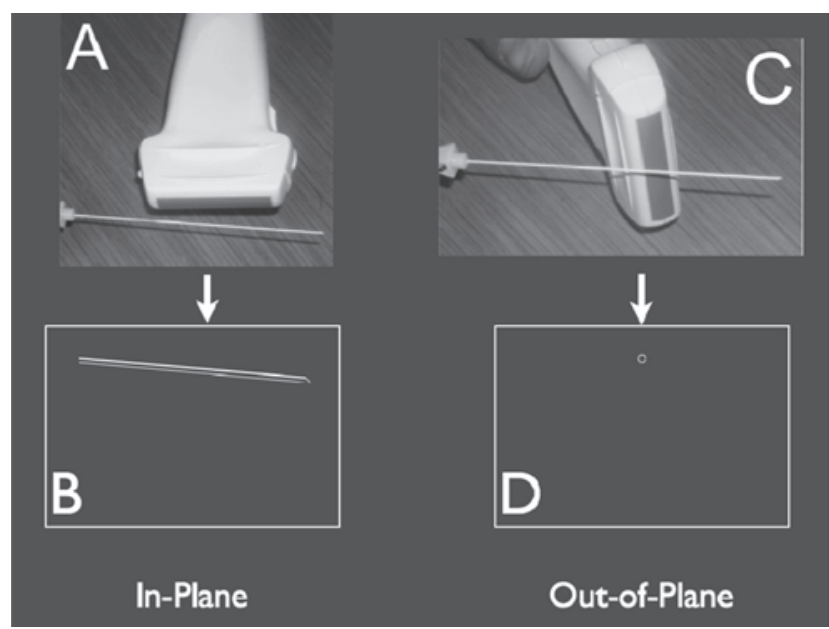

Figure 2 Two-dimensional ultrasound imaging during needle advancement. With the in-plane approach $\mathbf{A}$ ). the needle is visualized in its long axis B). In the out-of-plane approach $\mathbf{C}$ ), a limited view of the needle is generated secondary to the needle being imaged in short axis $\mathbf{D}$ ).

ultrasound guided regional block (see Table 1). A key aspect of these 10 steps includes stressing the importance of using the real time image to diagnose an intravascular injection or an intra-neuronal injection (Figure 3).

Research suggests that a major quality threat in the performance of UGRA is confusion by the operator regarding the orientation of structures visualized on the ultrasound screen with actual surface anatomy of the patient (Figure 4). It follows that the ASRA-ESRA Joint Committee has recommended a standardized and simple process for determining patient and screen orientation. For patients in any position, the operator states that screen left represents a defined anatomical aspect of the patient (eg, cephalad). To confirm this, the primary operator applies pressure with a finger at this defined site. A corresponding indentation should be visualized on the left aspect of the ultrasound screen.

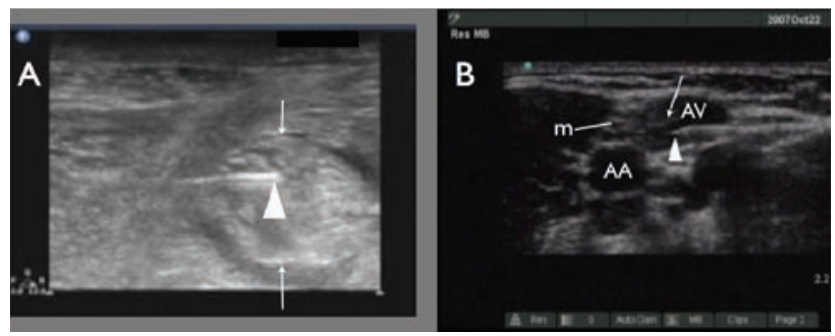

Figure 3 Inadvertent intra-neuronal needle placement during a popliteal sciatic nerve block A). The triangle indicates the needle tip inside the nerve and the arrows indicate the anterior and posterior aspects of the nerve. The inadvertent intra-vascular needle placement during an axillary nerve block B) $m$, median nerve, triangle reveals needle tip in axillary vein (AV);AA, axillary artery. Photo courtesy of Robert Weller, MD.

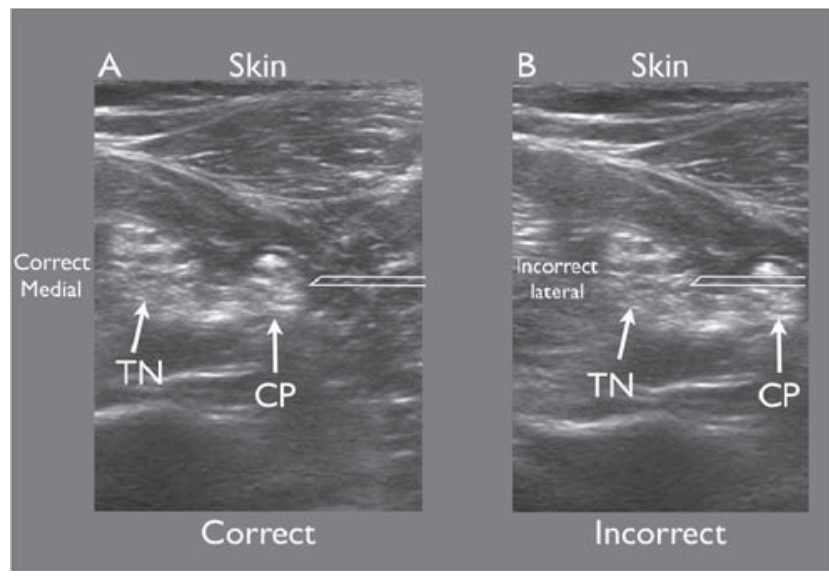

Figure 4 Right and left confusion. The operator correctly assumes that screen right is lateral and anticipates seeing the needle (simulated) entering from screen right A). However in B), the operator incorrectly assumes that screen left was lateral and was surprised to see the needle (simulated) entering from screen right. This was not appreciated until the needle had already penetrated the sciatic nerve.

If indentation occurs on screen right, then the operator must turn the transducer 180 degrees. Following such a correction, the operator should repeat skin pressure to confirm correct orientation.

\section{Case 2: It is 3 AM: The supraclavicular block} It is 3 AM and you are called to provide an anesthetic for an external fixation, irrigation, and debridement of an infected left proximal humerus in a women who sustained a closed fracture several days prior. This case may seem routine, but here is the catch: She is $186 \mathrm{~kg}$, IDDM, known difficult airway, on home oxygen, and has ischemic cardiomyopathy (EF 20\%), and her troponins are rising. What is your plan?

This is the moment when your regional anesthesia skills can really earn their keep. The major question is, do you believe in your skills and the described techniques enough to attempt what may seem impossible? We believe that this patient can easily and effectively have the "spinal of the arm," generating complete surgical anesthetic conditions. This block is otherwise known as the supraclavicular nerve block. David Brown, MD, author of a leading American textbook in regional anesthesia, has been known to say: "If I was stuck on a desert island with a needle and one block to do, it would be a supraclavicular block!" 6

Ultrasound has aggressively rejuvenated this exciting block. In contrast to an axillary approach to the brachial plexus, at the level of the first rib, the neural elements (trunks to divisions) are concentrated in a small anatomical area. Further, if local anesthetic is exposed to the 
trunks (superior, middle, and inferior), the entire plexus will be blocked. Given that this block targets the brachial plexus at the level of the first rib, there is proximity to the pleura and subclavian vessels. Conventional approaches to performing a supraclavicular block using surface landmarks are limited based on the concern of pneumothorax. Further, the subclavian artery represents a large collateral target that can facilitate the possibility of local anesthetic toxicity and hematoma formation. Figure 5 reveals the set up and approach to performing a supraclavicular block. Figure 6 reveals the key ultrasound anatomy associated with the supraclavicular approach to the brachial plexus. The two key structures that need to be avoided with the needle are the subclavian artery and pleura. These structures are easily seen.

Case resolution: The patient was positioned in the sitting position to facilitate her ventilation. A supraclavicular block was performed with a $50 \mathrm{~mm}$ B-Bevel needle and $25 \mathrm{~mL}$ of $0.5 \%$ bupivacaine without additives. The needle was visualized and advanced using the in-plane approach. The local anesthetic was injected in three locations to obtain circumferential spread of local anesthetic around the neural elements (trunks to divisions) at the level of the supraclavicular fossa. The patient received $1 \mathrm{mg}$ of intravenous midazolam and $25 \mu \mathrm{g}$ of intravenous fentanyl for the block. She required no additional sedation for the surgical procedure, consisting of an irrigation, debridement, and external fixator application. Following the procedure the patient was returned to the cardiac care unit with

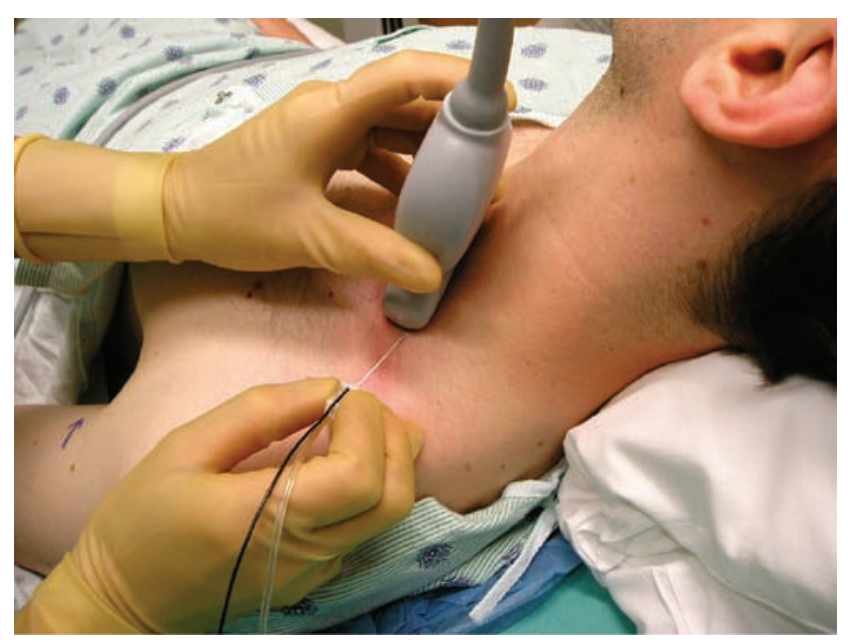

Figure $\mathbf{5}$ The set up for performing an ultrasound guided supraclavicular brachial plexus block (BP). The ultrasound transducer is positioned adjacent to the upper border of the clavicle and the image is then optimized using the PART maneuvers (also see Figure 23). Needle advancement is from a lateral to medial direction utilizing an in-plane approach.

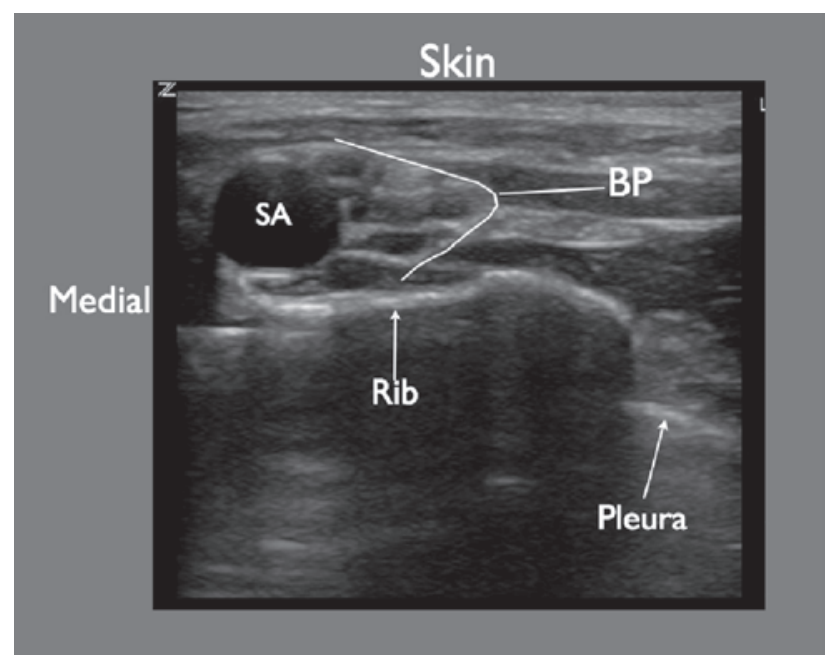

Figure 6 Ultrasound imaging of the supraclavicular brachial plexus block. Structures visualized here include: SA, subclavian artery; BP, brachial plexus. The pleura appears as a hyperechoic white line. The pleura appears to disappear medially as it is blocked by a large drop out shadow generated by the first rib.

nasal oxygen. She returned to the operating room three additional times for irrigations and final fixation. She had a supraclavicular block each time. It should be noted that other nerve block options were considered for this case, such as an infraclavicular approach. However, given her massive obesity, and, therefore, extreme depth to her infraclavicular brachial plexus, we opted to avoid this technique.

\section{Case 3: Increasing your armamentarium}

Your orthopedic surgical colleague is increasing his volume of total shoulder arthroplasties. You are interested in expanding your services to include continuous interscalene blocks. You have reviewed a posterior landmark based technique that has been advocated by a leader in regional anesthesia. ${ }^{7}$ However, the idea of inserting a 17 gauge needle through the posterior aspect of the neck to contact a cervical transverse process is rather unappealing to you. You wonder if there is a technique that can capitalize on the convenience of the posterior approach, but use ultrasound to target the roots of the brachial plexus directly without bony contact?

A technique has recently been described. ${ }^{8}$ The patient is placed in the lateral decubitis position with the operative side up. The ultrasound transducer is positioned on the neck generating a standard short-axis image of the roots of the brachial plexus (Figure 7). The roots of the brachial plexus will appear as distinct round to oval hypoechoic structures (Figure 8). The needle should be inserted approximately $2 \mathrm{~cm}$ from the edge of the transducer. Following subcutaneous infiltration of local anesthetic, A 17 or 18 gauge Tuohy needle is advanced utilizing the in-plane needle 




Figure 7 Demonstration of the set up for placement of an ultrasound guided interscalene catheter utilizing a posterior approach. The patient is placed in a latera decubitus position and the transducer is stabilized utilizing a mechanical stabilizing device. The Tuohy needle enters the skin approximately $2 \mathrm{~cm}$ from the transducer and advanced with an in-plane approach.

insertion approach. The entire length of the needle should remain parallel to the faceplate of the transducer, thus assuring full and unequivocal needle visualization. The needle is advanced until it enters the interscalene groove between the fifth and sixth ventral cervical nerve roots. After a negative aspiration is confirmed, 1 to $3 \mathrm{~mL}$ test dose of local anesthetic or D5W is injected slowly to confirm that the Touhy needle has penetrated the appropriate fascial layers. A total of 10 to $15 \mathrm{~mL}$ of the same solution is then injected slowly while visualizing the solution surrounding the roots of the brachial plexus. A catheter is subsequently advanced 2 to $4 \mathrm{~cm}$ past the tip of the needle (Figure 9). When the catheter exits the needle tip, it should be transiently visualized

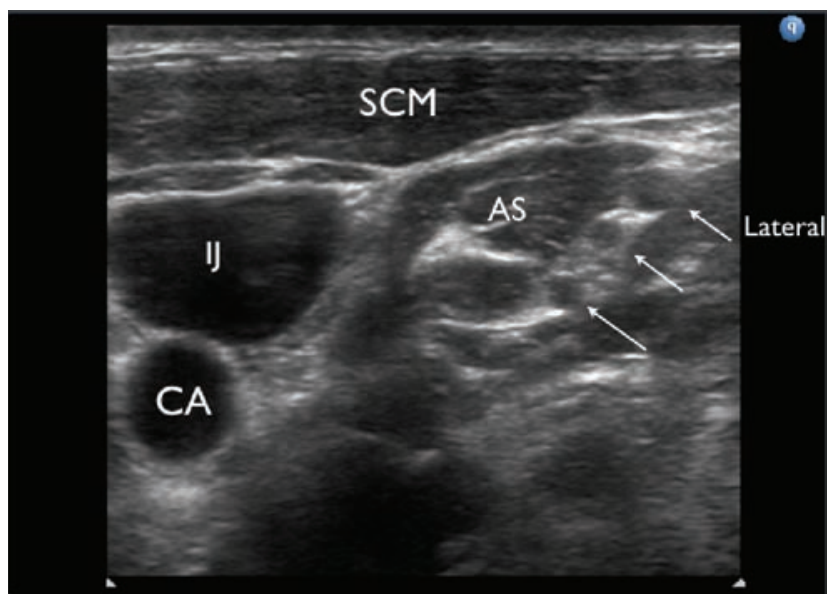

Figure 8 Ultrasound appearance of the roots of the brachial plexus. AS, anterior scalene muscle; IJ, internal jugular vein; CA, carotid artery; SCM, sternocleidomastoid muscle. Arrows refer to the 3 hypoechoic circles believed to be the roots of the brachial plexus.

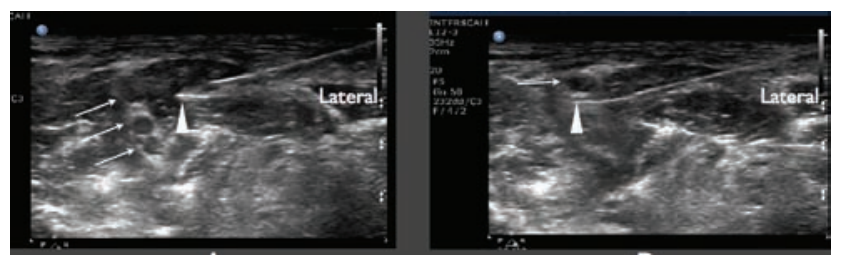

Figure 9 Tuohy needle advancement during an ultrasound guided interscalene catheter utilizing a posterior approach. In $\mathbf{A}$ ). the needle tip is seen advancing between the C5 and $\mathrm{C} 6$ nerve roots. The catheter is subsequently advanced and is seen exiting the Tuohy needle between the $\mathrm{C} 5$ and $\mathrm{C} 6$ nerve roots $\mathbf{B}$ ). Triangles refer to the needle tip in $\mathbf{A}$ ) and the catheter exiting the needle in $\mathbf{B}$ ). The arrows point to the roots of the brachial plexus in both $(\mathbf{A})$ and $(\mathbf{B})$.

as it defines its course either proximally or distally within the sheath. Figure 10 demonstrates what happens if the catheter meets resistance secondary to one of the scalene muscles. In this particular situation, simply retract the catheter into the needle, turn the bevel 90 degrees and re-thread. Following successful catheter advancement, the Tuohy needle is then removed. To confirm that the catheter tip is correctly positioned in the interscalene groove, additional test solution can be injected through the catheter. The spread of local anesthetic should have similar intra-sheath characteristics as for the needle injection. Visualizing the injection through the catheter is a critical quality maneuver, as it will be the catheter, not the needle that will be responsible for long-term analgesia. The catheter site is then dried, being careful to remove all the ultrasound gel. The catheter is subsequently secured to the skin with an adhesive sterile transparent dressing. It should be noted that for continuous imaging during this block, including during catheter advancement, two individuals would likely be needed, with one individual holding the transducer. Alternatively, there are mechanical devices that act as a third arm

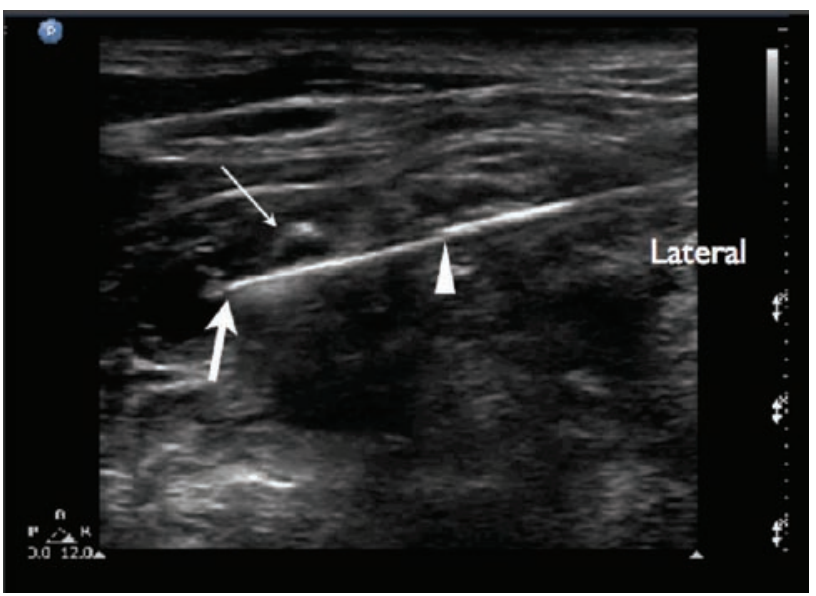

Figure 10 Demonstration of a catheter meeting resistance as it makes contact with the anterior scalene muscle.Triangle points to the needle tip. Large arrow points to the catheter tip as it makes contact with the anterior scalene muscle. Small arrow points to the $C 5$ nerve root. 
to hold the transducer, thus making the procedure essentially a one-person task.

Case conclusion: The catheter is placed in your first patient. The original bolus of $15 \mathrm{~mL}$ of $0.5 \%$ ropivacaine and then an infusion of $8 \mathrm{~mL} / \mathrm{h}$ resulted in pain scores of less than 2 for the duration of the hospital stay. Physical therapy was effective and the patient was discharged in the evening of post-operative day 2 after successful removal of the catheter and transition to oral pain control.

\section{Case 4: Rescue block}

Your partner performs a trans-arterial axillary for an open internal fixation of a radial head fracture in a $70 \mathrm{~kg}$ female with severe rheumatoid arthritis and known C1-C2 Involvement and subluxation. The nerve block misses both the median and musculocutaneous (lateral antecubital cutaneous) nerve distributions, despite using $30 \mathrm{~mL}$ of $0.2 \%$ lidocaine with 1/200 k of epinephrine. What do you do now? Does the patient need a general anesthetic?

Ultrasound allows us to successfully salvage a failed proximal brachial plexus block. Essentially, the median, ulnar, radial, and musculocutaneous nerves can be imaged and targeted at several locations at and distal to the axillary region. These have been described in some detail. ${ }^{9,10}$

The musculocutaneous nerve (MCN) block can be done with the arm abducted on a support table (Figure 11). The axillary region is scanned. The axillary artery is identified as a hypoechoic palatial circle and the transducer is then moved towards the biceps muscle. We usually target the MCN (in its short-axis) as it lies between the corocobrachialis and biceps muscles. This hyperechoic nerve is extremely easy to visualize because of the

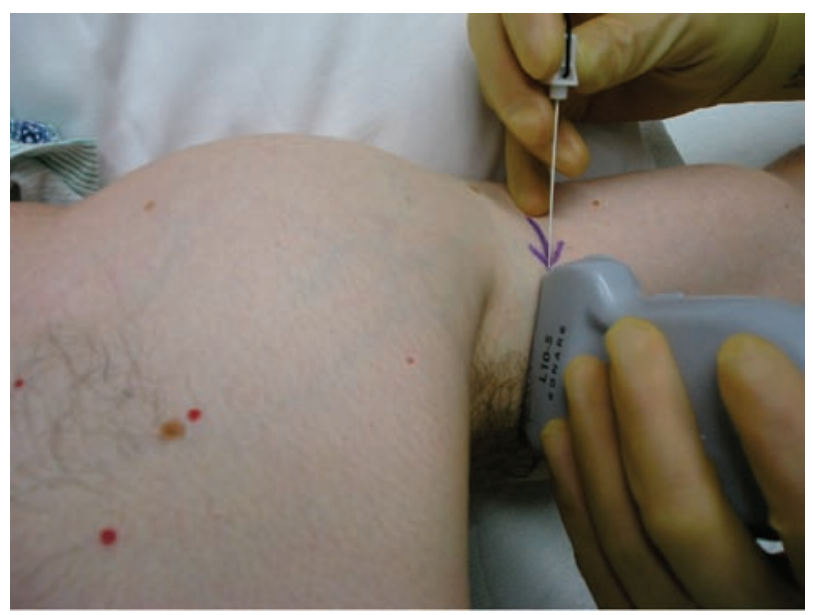

Figure I I The set up for an ultrasound guided musculocutaneous nerve (MCN) block. The arm is abducted on a support table.After a cross sectional view of the MCN, the needle enters the skin utilizing an in-plane approach. surrounding hypoechoic muscle, generating a distinct interface effect (Figure 12). The needle is inserted using the in-plane approach. Remember, you are limited regarding the amount of local anesthetic that can safely be utilized given the "large" dose from the original injection. It turns out that 2 to $3 \mathrm{~mL}$ is all that is needed to effectively block this nerve. Figure 13 demonstrates what $2 \mathrm{~mL}$ can accomplish.

The median nerve can be traced distally from the axilla to the wrist crease. We prefer to target the median nerve in the distal 1/3 of the forearm (Figure 14). In its short axis, the median nerve appears as a hyperechoic circle with internal hypoechoic fascicles (Figure 1B and 1D). Since this nerve is usually less than $1 \mathrm{~cm}$ deep, high frequency ultrasound can be utilized providing amazing detail. Again, the in-plane technique is utilized and 2 to $3 \mathrm{~mL}$ is deposited circumferentially around the nerve.

Case conclusion: With a total of $6 \mathrm{~mL}(0.5 \%$ ropivacaine $)$, both nerves were successfully blocked and the patient underwent the proposed procedure with conscious sedation. Her airway and neck were not manipulated. She went home 1 hour post surgery.

\section{Lower extremity}

\section{Case 5: You really want to perform a spinal, but you cannot}

A 71-year-old male presents for a left revision of an above the knee amputation. He has a history of hypertension, diabetes mellitus, coronary artery disease, and an ischemic cardiomyopathy with an ejection fraction of $15 \%$. He is on anticoagulation therapy consisting of clopidogrel and aspirin. Given his morbid medical conditions you decide a regional anesthetic would be best. What techniques are

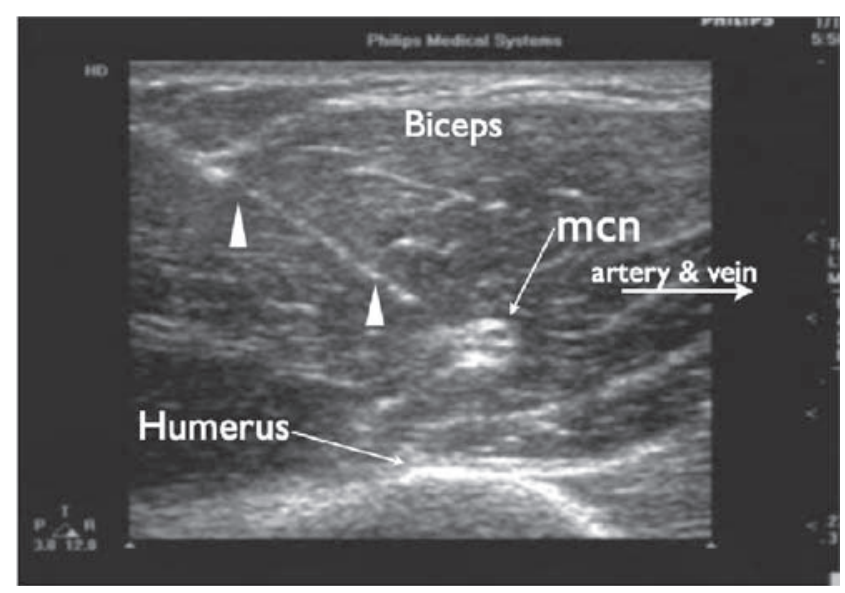

Figure I 2 Ultrasound image of a cross sectional view of the musculocutaneous nerve. Needle advancement is with an in-plane approach.Triangles indicate the needle. $\mathrm{mcn}$, musculocutaneous nerve. 


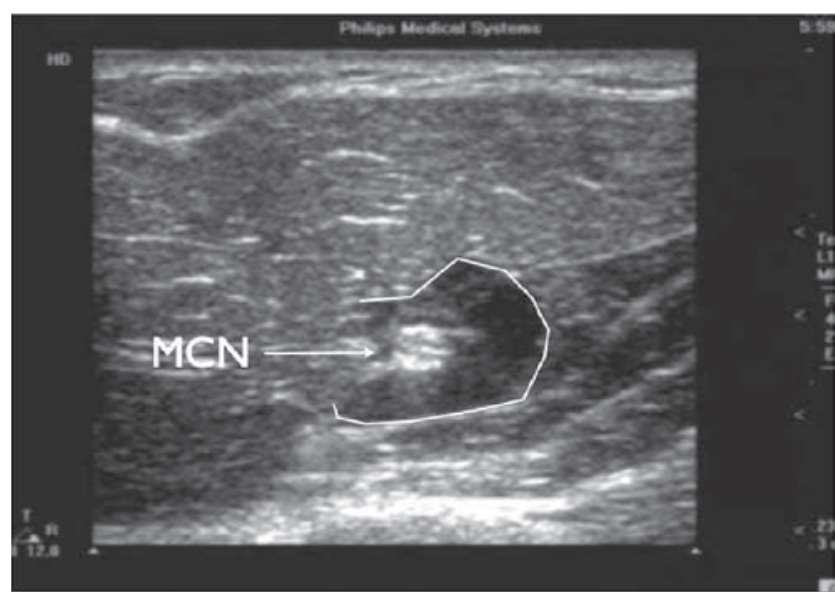

Figure 13 The musculocutaneous nerve after depositing $3 \mathrm{~mL}$ of local anesthetic. The local anesthetic is outlined by the white line.

at your disposal? What is the evidence to support your approach? How will you proceed?

Patients such as the one described here provide numerous challenges for the anesthesia provider. Regional anesthesia would be an attractive choice, as it may minimize the inflammatory effects of general anesthesia and pain. Given his anticoagulation status, neuraxial anesthesia is an unattractive option. A peripheral nerve blockade of his left lower extremity would be ideal. This could be accomplished with a combined lumbar plexus and sciatic nerve blocks. However, even though a lumbar plexus block would adequately anesthetize the lateral femoral cutaneous, femoral, and obturator nerves there would still be a significant risk of

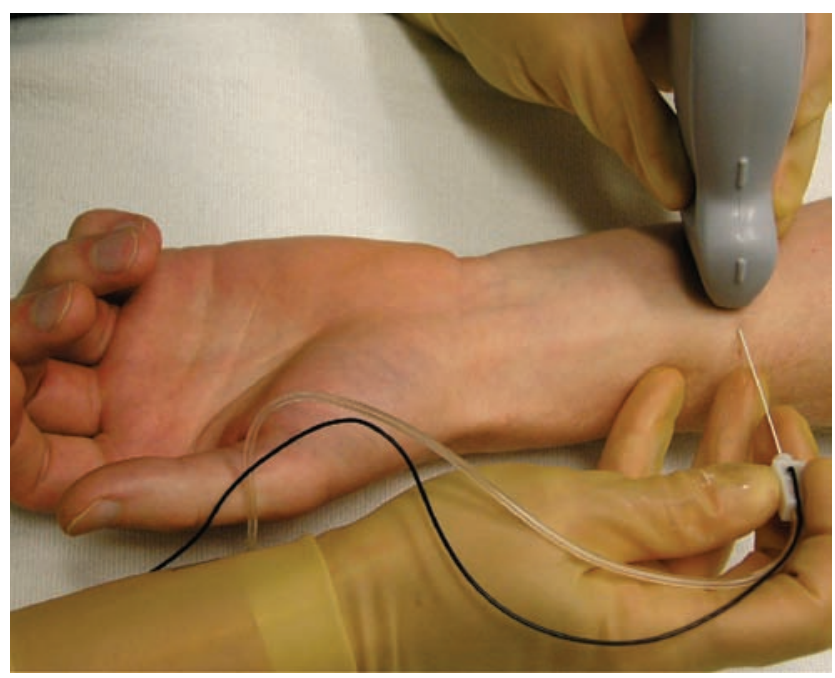

Figure 14 The set up for an ultrasound guided median nerve block in the distal I/3 of the forearm. The forearm is rested on a padded table and the needle enters utilizing and in-plane approach. a retroperitoneal bleed. Additional options would include the placement of a femoral nerve catheter threaded proximally such that an effective lumbar plexus block is obtained. Alternatively, the femoral, obturator, and lateral femoral cutaneous nerves could be blocked separately.

Traditional superficial landmark and nerve stimulation techniques have several limitations. What is obvious in the present case is that objective findings of a motor response to nerve stimulation would be difficult if not impossible with a preexisting above the knee amputation. Ultrasound guided nerve blocks can overcome this limitation as neither a quadriceps contraction nor plantar flexion of the foot is required. The fundamental objective of an ultrasound guided peripheral nerve block is to visualize effective perineural spread of local anesthetic in real time. Other similar situations include patients with splinted extremities, abnormal surface anatomy, or preexisting neuronal dysfunction. ${ }^{11-13}$

In several recent studies, ultrasound has demonstrated an inherent false negative rate of nerve stimulation. In other words, despite needle to nerve contact, no motor response is elicited. During an ultrasound guided axillary nerve block at a current threshold of less $0.5 \mathrm{~mA}$, Perlas and colleagues found that despite needle to nerve contact only $75 \%$ had a motor response. ${ }^{14}$ Similarly, during supraclavicular nerve blocks, Beach and colleagues reported that only $87 \%$ of patients had a motor response to nerve stimulation at $0.5 \mathrm{~mA} .{ }^{15}$ More recently, Sinha et al reported that only $42 \%$ of patients had a motor response at less than $0.5 \mathrm{~mA}$ when the needle was placed between what was presumed to be the $\mathrm{C} 5$ and $\mathrm{C} 6$ nerve roots. However, all patients had a complete motor and sensory block. ${ }^{16}$ Results such as these would suggest that when nerve stimulation alone is used, multiple needle attempts and repositioning would be performed unnecessarily.

Patients with diabetes mellitus are known to have underlying neurological dysfunction involving a progressive impairment of sensory and motor function. ${ }^{17}$ There is a progressive decrease in nerve conduction velocity and amplitude in sensory and motor nerves. ${ }^{18} \mathrm{~A}$ recent report by Sites et al suggests a reduced effectiveness in localizing nerves with standard nerve stimulation techniques in patients with diabetes mellitus. In this report a current as high as $2.4 \mathrm{~mA}$ was required when applied to the sciatic nerve before a motor response was achieved. ${ }^{19}$ Rigaud et al demonstrated in an animal model with induced acute hyperglycemia that a low threshold stimulation of $0.5 \mathrm{~mA}$ uniformly resulted in intraneural injections. ${ }^{20}$ 
Marhofer and coworkers demonstrated that ultrasound had an advantage over nerve stimulation techniques by improving the quality of sensory blockade in 3-in-1 blocks while reducing the onset time and amount of local anesthetic used. ${ }^{21,22}$ However, our preference would be placement of an ultrasound guided femoral nerve catheter threaded near the lumbar plexus. Anatomic dissections suggest it would be impossible to block the obturator nerve with a single injection femoral nerve block (see Figure 15). In fact, Capdevila et al demonstrated that a reliable obturator nerve block occurred when catheters were threaded to the lumbar plexus. ${ }^{23}$ Although we have had great success with this approach, current evidence would suggest that the catheter reaches the lumbar plexus only $23 \%$ to $40 \%$ of the time. ${ }^{23}$ When a lumbar plexus catheter is our intent, we will obtain a radiographic film of these catheters with $3 \mathrm{~mL}$ of contrast dye to confirm location. Depending on catheter placement, we may supplement the lateral femoral cutaneous and/or obturator nerve using ultrasound guidance as these techniques have recently been described. ${ }^{24-27}$

Chan et al demonstrated the usefulness of ultrasound imaging in identifying the sciatic nerve in the gluteal, infragluteal, and proximal thigh region. ${ }^{28}$ In a prospective randomized trial Domingo-Triadó et al ${ }^{29}$ compared mid-femoral sciatic nerve blockade using ultrasound plus nerve stimulator to nerve stimulator alone. Block failure was similar between the ultrasound guided and nerve stimulationguided groups (3\% vs 6\%). Ultrasound guidance, however, reduced the number of attempts required to localize the



Figure I5 Anatomic dissection showing the relationship of the obturator nerve to the femoral artery and femoral nerve. Notice the distance and the barriers for local anesthetic spread to the obturator nerve when performing a single injection femoral nerve block. FA, femoral artery; FN, femoral nerve; ON, obturator nerve. sciatic nerve as well as the number of patients who had partial or no sensory block after 1 h (3 vs 29\%). More recently Perlas et $\mathrm{al}^{2}$ showed that ultrasound guidance improved the success rate of sciatic nerve block performed in the popliteal fossa when compared to nerve stimulation alone $(89.2 \%$ vs $60.6 \%, \mathrm{p}=0.005)$.

Case resolution: This patient was positioned in a supine position. The femoral nerve was visualized with a linear transducer scanning at $10 \mathrm{MHz}$. Lateral to the femoral nerve the fascia iliaca was visualized. A Touhy needle was placed with the out-of plane technique lateral to the femoral nerve (Figure 16). Fifteen milliliters of $0.5 \%$ ropivacaine was injected while visualizing the spread of local anesthetic under the fascia iliaca (Figure 17). Subsequently a catheter was threaded $12 \mathrm{~cm}$ until resistance was met. A radiograph of the catheter was then obtained which confirmed that the tip was at the level of the lumbar plexus (Figure 18). Ten milliliters of $0.5 \%$ ropivacaine was injected to anesthetize the lateral femoral cutaneous and obturator nerves. The patient was then positioned in a lateral decubitus position with a slight forward tilt for a sub-gluteal sciatic nerve block (Figure 19). A low frequency curved array transducer scanning at $5 \mathrm{MHz}$ was used to identify the sciatic nerve (Figure 20). Using the in-plane approach, a subgluteal sciatic nerve block was performed with a $100 \mathrm{~mm}$ B-Bevel needle and $25 \mathrm{~mL}$ of $0.5 \%$ ropivacaine. The patient then proceeded for revision of his AKA under conscious sedation. It should be noted that the decision to obtain an x-ray or live fluoroscopy to confirm catheter placement represents an addition monetary and time cost. We found these studies to be immensely helpful during our "learning curve" in terms of

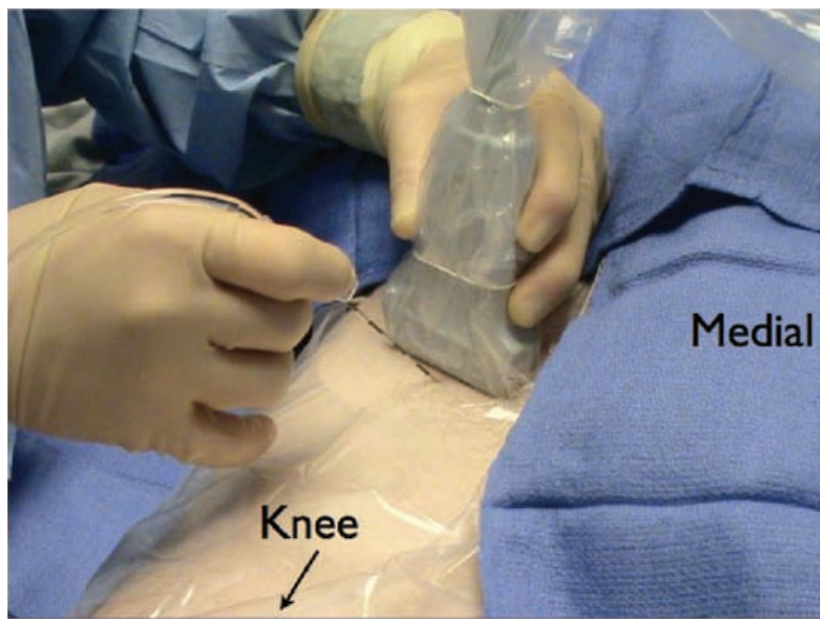

Figure 16 The set up when placing an ultrasound guided femoral nerve catheter. The patient is positioned supine and the transducer is aligned with the inguinal crease. After image optimization, the needle enters the skin utilizing the out-of-plane approach. 




Figure 17 The femoral nerve after local anesthetic injection. The local anesthetic appears to be spreading beneath the fascia iliaca and in a circumferentially around the femoral nerve. Triangle indicates the fascia iliaca. FN, femoral nerve; FA, femoral artery.

appreciating what needle maneuvers and tissue resistances predicted success and failure.

\section{Case 6:"I don't want to vomit"}

A 44-year-old male presents for an open reduction and internal fixation of his right ankle. He informs you that with his previous surgeries he had retractable nausea and vomiting that led to several unplanned hospital admissions. He wishes to be "awake" for surgery. You get your surgeon to agree not to use a thigh tourniquet. You discuss performing a single injection

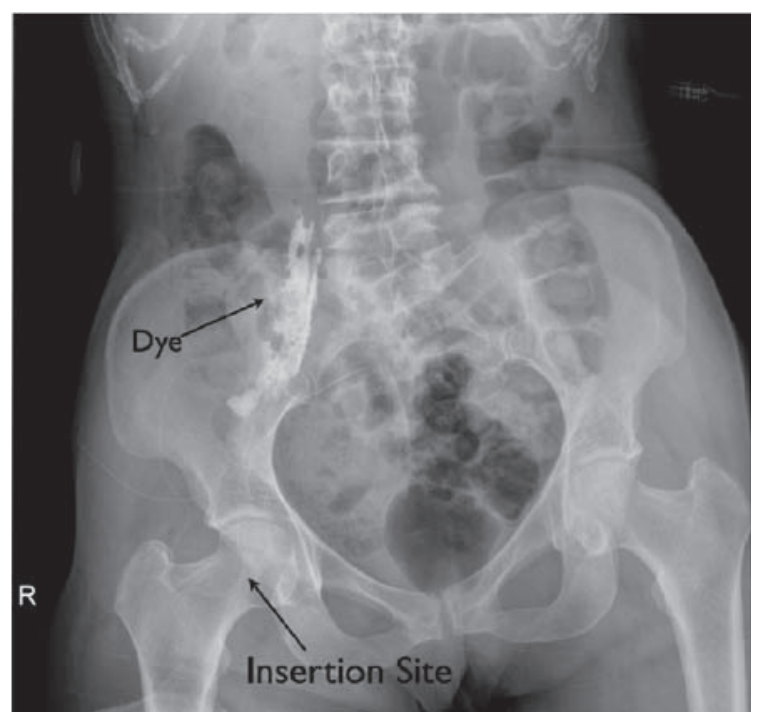

Figure $18 \mathrm{~A}$ femoral nerve catheter is placed and advanced to the lumbar plexus. This is confirmed with a radiograph of the catheter after injecting $3 \mathrm{~mL}$ of contrast dye.

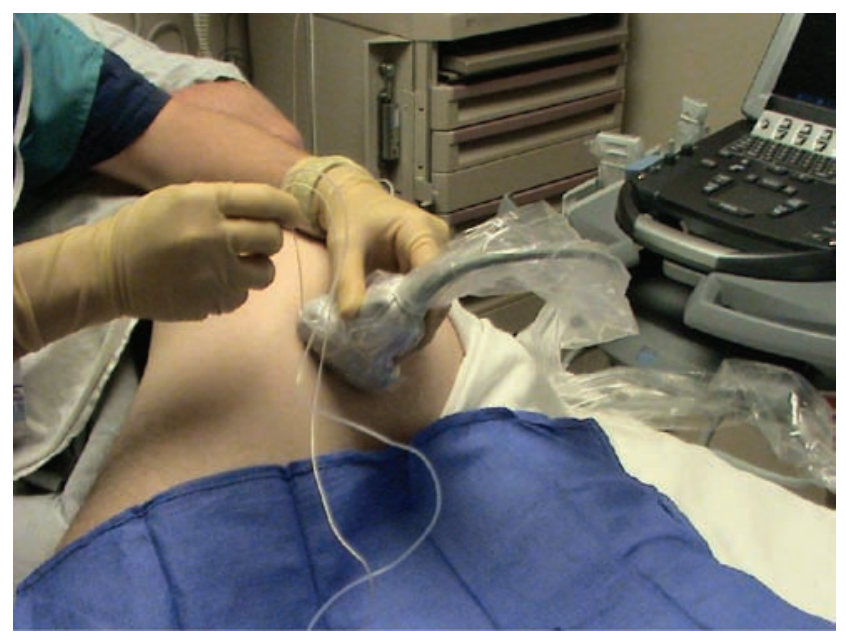

Figure 19 The set up for a single injection infragluteal sciatic nerve block. The patient is placed in a lateral position with forward pelvic tilt. A low frequency $(5-2 \mathrm{MHz})$ curved array transducer is placed on a distinct grove that can be palpated lateral to the attachment of the bicep femoris muscle on the ischial tuberosity. The image generated can be seen in Figure 20.

popliteal sciatic nerve block combined with a mid-thigh saphenous nerve block for surgical anesthesia.

Blocking the sciatic nerve in the popliteal fossa is easy because it is a relatively superficial structure surrounded by adipose tissue (Figure 21), creating a stark echo interface (Figure 22). The sciatic nerve appears distinctly hyperechoic, whereas the adipose tissue is hypoechoic. Thinner patients with less adipose tissue in their popliteal fossa present more of an imaging challenge because the sciatic nerve may not be easily delineated from the surrounding muscle.

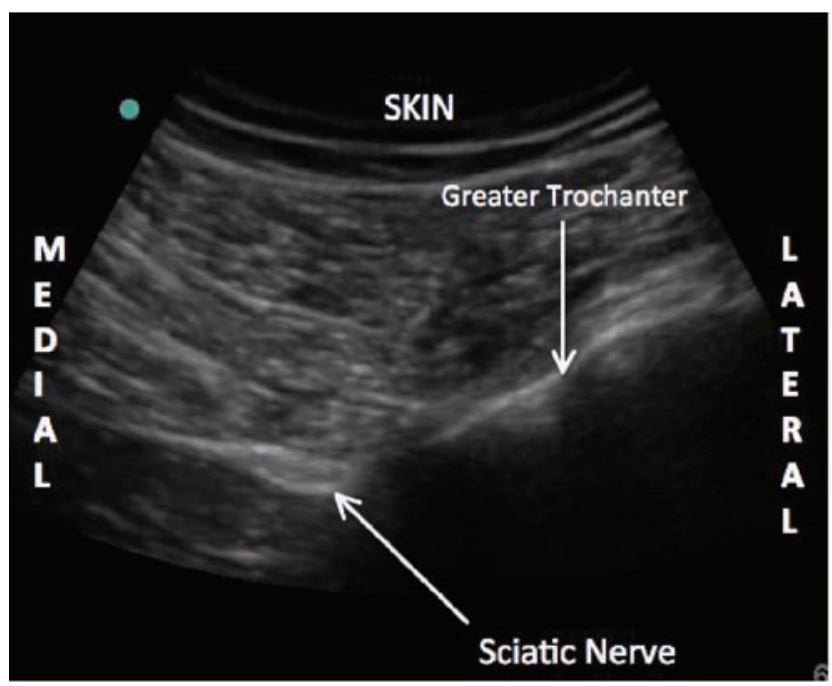

Figure 20 Ultrasound image of the infragluteal sciatic nerve. Notice the relationship of the sciatic nerve relative to the greater trochanter that is generated utilizing this imaging plane. 


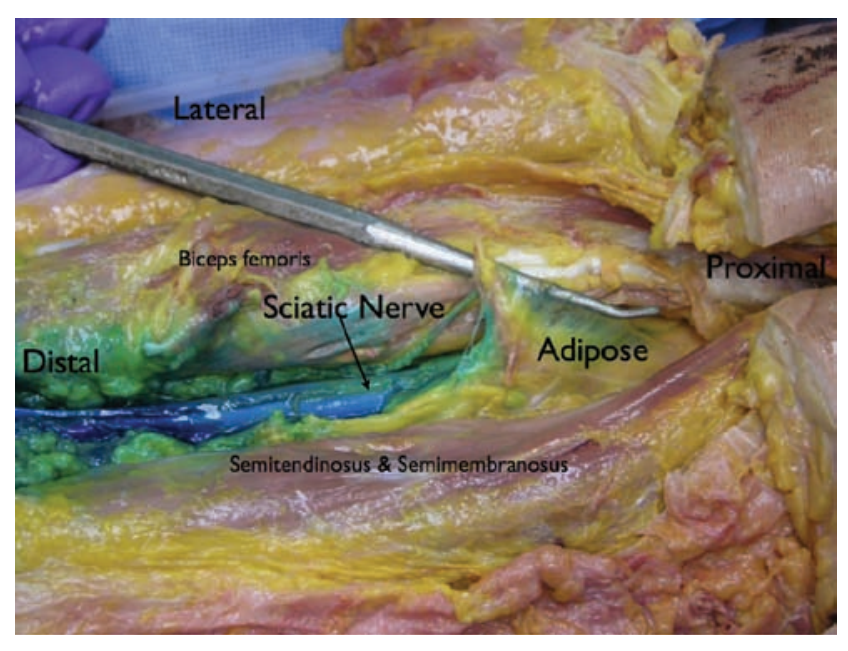

Figure 2I Anatomic dissection showing the sciatic nerve in the popliteal region. Notice the adipose tissue surrounding the nerve in this region.

When ultrasound imaging any target structure, the operator can perform four basic transducer maneuvers that to optimize the image quality. 5 These are referred to as the "PART" maneuvers which include: pressure, alignment, rotation, and tilting (Figure 23). In the case above, the patient is positioned supine and the extremity to be blocked is elevated on a support structure (Figure 24). The transducer is placed in the popliteal fossa and the popliteal artery is visualized. The hyperechoic tibial nerve can be seen posterior to the popliteal artery. Pressure (P) is applied to ensure contact between the transducer and the skin and to minimize the distance from the transducer to the neural structures. Next, the transducer is tilted $(\mathrm{T})$ in order to maximize the amount of ultrasound

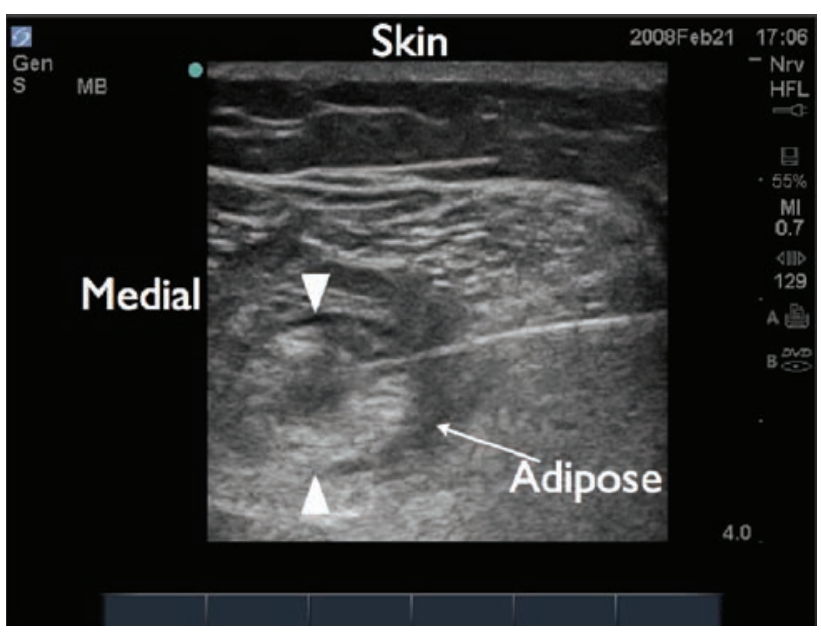

Figure 22 Ultrasound image of the sciatic nerve in the popliteal region. The adipose tissue surrounding the nerve is hypoechoic relative to the more echogenic sciatic nerve. This allows for better visualization of the nerve.
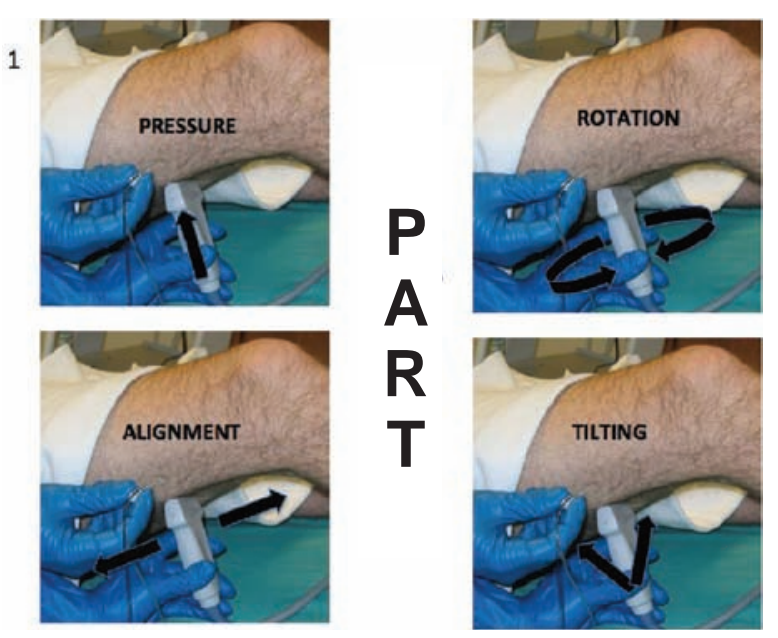

Figure 23 The "PART" maneuvers are performed to optimize ultrasound imaging of neural structures. The goal of these maneuvers is to maximize reflection back to the transducer and minimize refraction (bending of the ultrasound beam away from the transducer).

energy that is reflected at the nerve-adipose interface. Figure 25 demonstrates how the tilting maneuver can optimize the image of the sciatic nerve. Rotating $(\mathrm{R})$ the transducer clockwise or counterclockwise can then be performed to achieve a true short-axis view of the tibial nerve. This will minimize a diagonal or longitudinal imaging plane through the nerve. The transducer is then physically moved cephalad (Alignment) until the common peroneal nerve is visualized entering from a lateral to medial direction to join the tibial nerve. "Alignment" (A) is the act of physically moving the transducer without altering the pressure, tilt, or rotation.

It is essential to visualize the needle in its entirety when performing ultrasound guided nerve blocks. At the very least

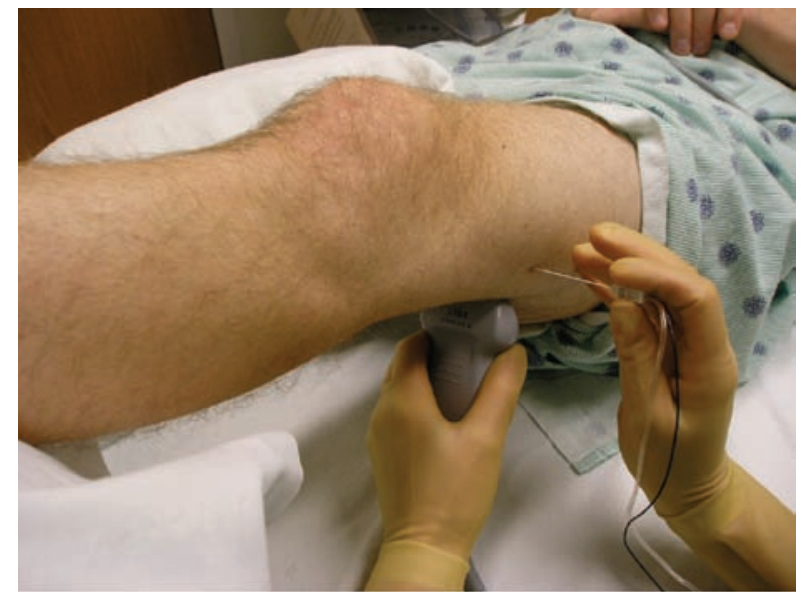

Figure 24 The set up for an ultrasound guided popliteal sciatic block. The patient is supine with the extremity elevated. The transducer is first placed in the popliteal region and then moved in a cephalad direction until there is an optimal view of the common peroneal and tibial nerves joining to form the sciatic nerve. After measuring the depth of the sciatic nerve, the needle is advanced using an in-plane approach. 


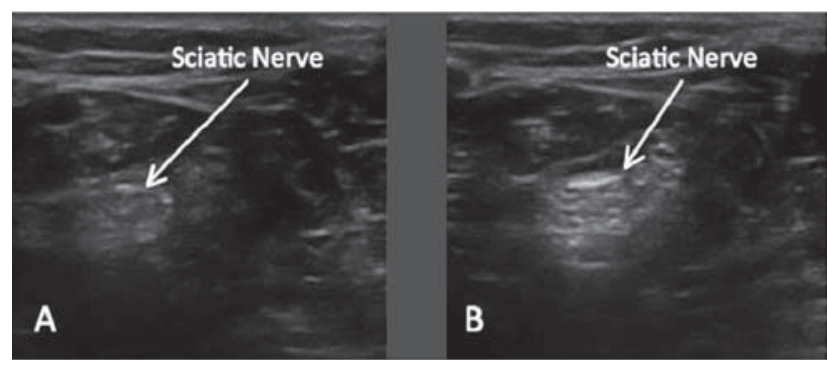

Figure 25 Demonstration of the tilting maneuver during the PART maneuvers. The sciatic nerve is seen in A). however, when the transducer is tilted slightly, a clearer image of the nerve is appreciated $\mathbf{B}$ ). This simple maneuver allows for more reflection and less refraction of the beam as it contacts the sciatic nerve.

the tip of the needle must be identified prior to injection. Maintaining needle visualization during advancement is essential to prevent complications. Sites et al ${ }^{30}$ studied novice behavior during 1-month regional anesthesia rotations. One of the most common persistent errors was failure to visualize the needle during advancement. Other common errors were inadequate equipment preparation and unintentional probe movement. Unintentional probe movement can be minimized if the operator braces his/her hand against either the patient or the bed. Notice in Figure 24 that the scanning arm is braced against the bed. This will also help to minimize fatigue.

A distinct advantage of ultrasound guided nerve blocks is that it allows real time visualization of the spread of local anesthetic. When performing a popliteal sciatic nerve block, the needle enters from the lateral aspect of the thigh and the common peroneal nerve first. If all the local anesthetic is deposited next to the common peroneal nerve then the tibial nerve will likely be spared. A "doughnut sign" in which local anesthetic circumferentially surrounds the nerve is likely the optimal end point when performing a sciatic nerve block. ${ }^{31}$

The saphenous nerve is the terminal branch of the femoral nerve and must be blocked for complete anesthesia of the ankle. Our favorite approach to blocking the saphenous nerve is with a new proximal to mid-thigh approach. This review article represents the first description of such a technique. Here, the saphenous nerve lies in close proximity to the superficial femoral artery with the neurovascular structures confined by easy to identify thick fascial plane (Figures 26 and 27). Using the superficial femoral artery as a reference point and the in-plane technique, we deposit 5 to $10 \mathrm{~mL}$ of local anesthetic as a peri-arterial injection. Imaging of the saphenous nerve at this location is not necessary.

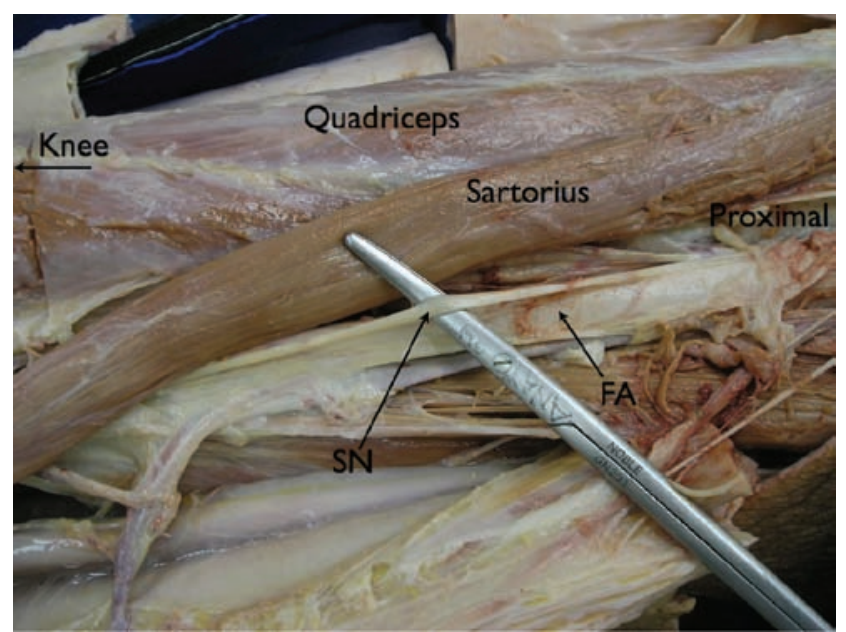

Figure 26 Anatomic dissection of the saphenous nerve at the mid-thigh level. The sartorius muscle has been retracted laterally. The nerve is appreciated lateral to the superficial femoral artery. SN, sciatic nerve; FA, femoral artery.

Case resolution: An ultrasound guided popliteal sciatic nerve block was performed with $25 \mathrm{~mL}$ of $0.5 \%$ bupivacaine utilizing an in-plane approach. A mid-thigh saphenous nerve block was also performed with $10 \mathrm{~mL}$ of $0.5 \%$ bupivacaine utilizing ultrasound and the in-plane approach. He had a successful surgical saphenous/sciatic nerve block. He refused sedation in the operating room and was discharged without nausea. It should be noted that the patient was presented the option of a spinal anesthetic instead of the nerve blocks. However, given the possibility of urinary retention, delayed discharge, and possible spinal headache, the patient opted against this anesthetic technique.

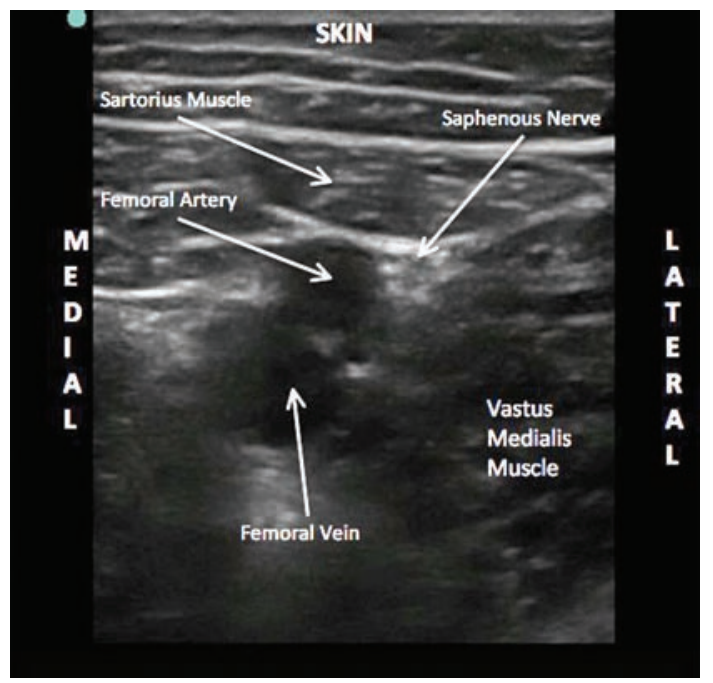

Figure $\mathbf{2 7}$ Ultrasound image of the saphenous nerve at the mid-thigh level. Notice the superficial femoral artery and saphenous nerve are lying deep to the sartorius muscle. The nerve lies in a superior-lateral position relative to the artery. 


\section{Case 7: You got to be kidding me, an ultrasound guided ankle block!}

A 64-year-old female with a history of obstructive sleep apnea and morbid obesity presents for a bunionectomy of her right and left great toe and pinning of her right 5th toe. The patient and the anesthetist agree that regional anesthesia with minimal sedation is the ideal anesthetic and she agreed to have bilateral ankle blocks.

The five nerves of the ankle involved when performing an ankle block are: deep peroneal, tibial, saphenous, sural, and superficial peroneal nerves. They cause minimal motor impairment and allow for ambulation. Although considered a basic nerve block, traditional techniques have highly variable success rates. ${ }^{32-34}$ Ultrasound guided ankle blocks are now commonly performed at our institution with great success and exemplify the creativity that ultrasound technology has allowed us to employ.

We first investigated the use of ultrasound for performing ankle blocks when a neurosurgeon at our institution was harvesting the sural nerve for diagnostic purposes. A known fact in the neurosurgery literature, but not in the anesthesia literature, is that the sural nerve lies adjacent to the lesser saphenous vein. We performed a prospective, double-blinded, volunteer study demonstrating that a peri-vascular technique utilizing the lesser saphenous vein as a reference point was superior to a traditional approach..$^{35}$ The tibial nerve ${ }^{36}$ and deep peroneal nerve are also amenable to ultrasound guidance.

Ergonomically it easiest start with the patient in a prone position. All nerves are targeted $1 \mathrm{~cm}$ proximal to the melleoli. Here the sural and tibial nerves are visualized (Figures 28 and 29). Both nerves can be approached utilizing

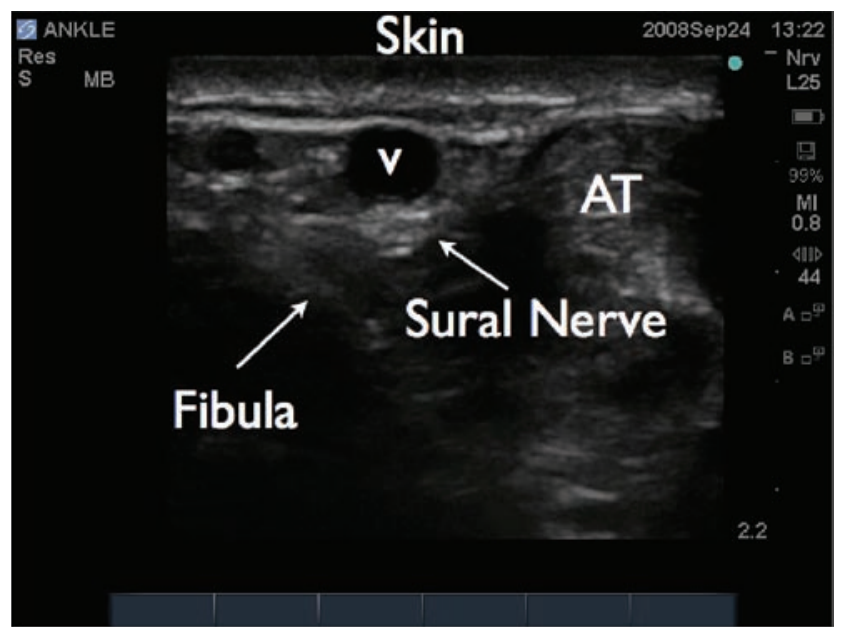

Figure 28 Ultrasound image of the sural nerve in the ankle. The lesser saphenous vein is identified $(\mathrm{V})$. The sural nerve lies adjacent (lateral, medial, or posterior) to this vein. AT, achilles tendon.

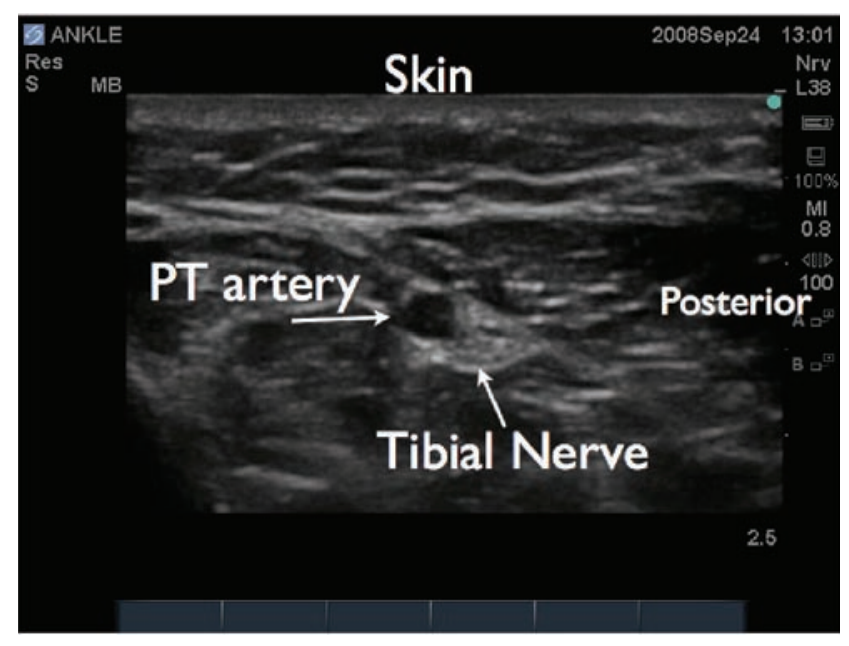

Figure 29 Ultrasound image of the tibial nerve in the ankle. The posterior tibial artery is seen (PT). The tibial nerve is seen posterior and lateral to the artery.

either an in-plane or out-of-plane technique (Figure 30). The patient is then turned supine. The deep peroneal nerve can be visualized on the dorsum of the ankle. It lies approximately 1 to $2 \mathrm{~cm}$ below the skin and lateral to the dorsalis pedis artery (Figure 31). It is approached utilizing an outof-plane technique (Figure 32). Finally, a skin wheal is raised extending from the medial to the lateral malleolus to anesthetize the saphenous and superficial peroneal nerves.

Case resolution: All 5 nerves were blocked on the right ankle and all but the sural nerve were blocked on the left ankle. The left sural nerve was spared given that the surgery did not involve its distribution. Three to five milliliters of $0.5 \%$ bupivacaine was used for each nerve depending on the

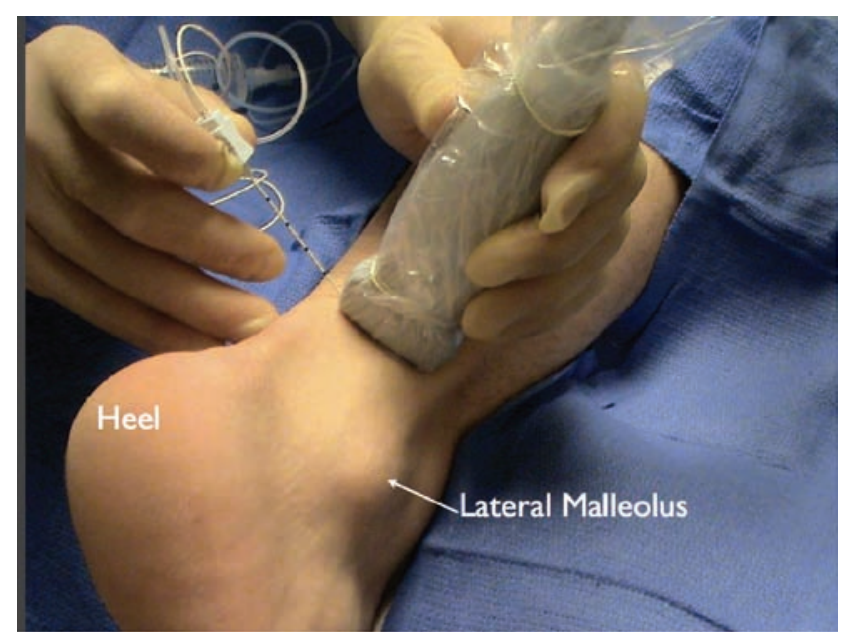

Figure $\mathbf{3 0}$ The set up for a sural nerve block at the ankle. The patient is placed in the prone position. The transducer is placed posterior and cephalad to the lateral malleolus. The nerve can be approached with either an in-plane or out-of-plane technique. 




Figure 3 I Ultrasound image of the deep peroneal nerve in the ankle. The dorsalis pedis artery is visualized first. The nerve can be appreciated just lateral to the artery.

spread of local anesthetic. In the end, bilateral ultrasound guided ankle blocks were performed with a total of $28 \mathrm{~mL}$ of $0.5 \%$ bupivacaine. After a successful surgical anesthetic with minimal sedation, the patient was comfortable and discharged home.

\section{Summary}

In summary, these case vignettes summarize important components of state of the art UGRA. Take home points should include:

1) Ultrasound allows for the real time evaluation of neural anatomy, needle position, and spread of local anesthesia.

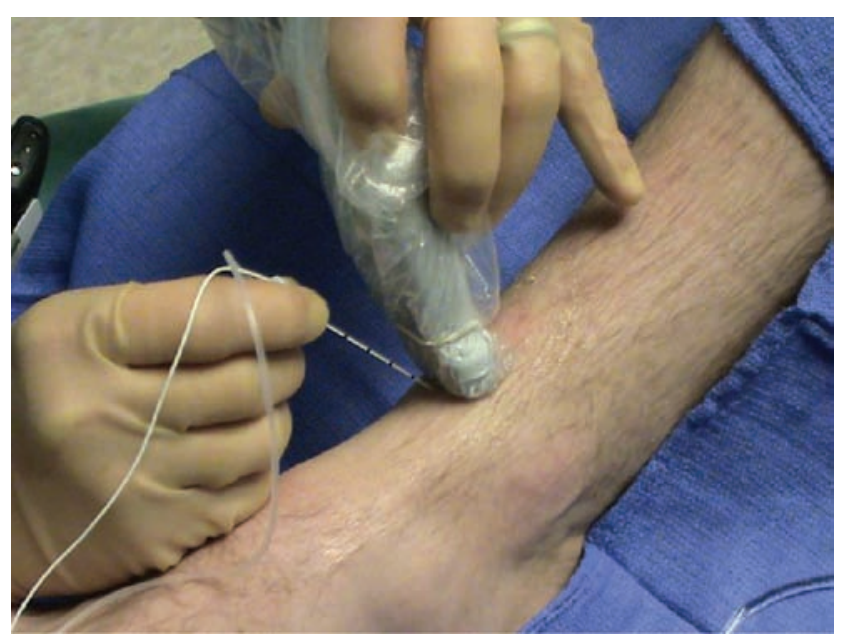

Figure 32 The set up for an ultrasound guided image of a deep peroneal nerve block. The patient is placed supine with the knee bent. The transducer can be placed at the ankle crease, slightly proximal, or slightly distal. The needle is advanced with an out-of-plane approach.
2) Challenging patients can be successfully approached with regional anesthesia.

3) New techniques are emerging for continuous blocks.

4) Nerves in the distal extremity can easily be blocked and imaged.

Future research will likely be directed toward applications of 3D imaging, defining the limitations of ultrasound technology, and how best to teach competencies associated with UGRA.

\section{Disclosures}

The authors have no conflicts of interest to disclose.

\section{References}

1. Kapral S, Greher M, Huber G, et al. Ultrasonographic guidance improves the success rate of interscalene brachial plexus blockade. Reg Anesth Pain Med. 2008;33:253-258.

2. Perlas A, Brull R, Chan VW, McCartney J, Nuica A, Abbas S. Ultrasound guidance improves the success of sciatic nerve block at the popliteal fossa. Reg Anesth Pain Med. 2008;33:259-265.

3. Chan VW, Perlas A, McCartney J, Brull R, Xu D, Abass S. Ultrasound guidance improves success rate of axillary brachial plexus block. Anesthesiology. 2007;106:992-996.

4. Williams SR, Chovinard P, Arcand G, et al. Ultrasound guidance speeds execution and improves the quality of supraclavicular block. Anesth Analg. 2003;97:1518-1523.

5. Sites B, Chan V, Neal J, et al. The American Society of Regional Anesthesia and Pain Medicine (ASRA) and the European Society of Regional Anaesthesia and Pain Therapy (ESRA) Joint Committee Recommendations for Education and Training in Ultrasound Guided Regional Anesthesia. Reg Anesth Pain Med. 2008. In Press.

6. Personal communication with David Brown, MD.

7. Boezaart AP. Patient-controlled interscalene analgesia after shoulder surgery: catheter insertion by the posterior approach. Anesth Analg. 2006;102:1902.

8. Antonakakis J, Sites B, Shiffrin J. Ultrasound guided posterior approach for the placement of a continuous interscalene catheter: a brief technical support. Reg Anesth Pain Med. 2008. In press.

9. Spence BC, Sites BD, Beach ML. Ultrasound guided musculocutaneous nerve block: a description of a novel technique. Reg Anesth Pain Med. 2005;30:198-201.

10. Macaire P, Singelyn F, Narchi P, Paqueron X. Ultrasound- or nerve stimulation-guided wrist blocks for carpal tunnel release: a randomized prospective comparative study. Reg Anesth Pain Med. 2008;33:363-368.

11. Plunkett AR, Brown DS, Rogers JM, Buckenmaier CC. 3rd. Supraclavicular continuous peripheral nerve block in a wounded soldier: when ultrasound is the only option. Br J Anaesth. 2006;97:715-717.

12. van Geffen GJ, Scheuer M, Muller A, et al. Ultrasound guided bilateral continuous sciatic nerve blocks with stimulating catheters for postoperative pain relief after bilateral lower limb amputations. Anaesthesia. 2006;61:1204-1207.

13. Assmann N, McCartney CJ, Tumber PS, Chan VW. Ultrasound guidance for brachial plexus localization and catheter insertion after complete forearm amputation. Reg Anesth Pain Med. 2007;32:93.

14. Perlas A, Niazi A, McCartney $\mathrm{C}$, et al. The sensitivity of motor response to nerve stimulation and paresthesia for nerve localization as evaluated by ultrasound. Reg Anesth Pain Med. 2006;31:445-450.

15. Beach ML, Sites BD, GallagherJD. Use of a nerve stimulator does not improve the efficacy of ultrasound guided supraclavicular nerve blocks. J Clin Anesth. 2006;18:580-584. 
16. Sinha SK, Abrams JH, Weller RS. Ultrasound guided interscalene needle placement produces successful anesthesia regardless of motor stimulation above or below $0.5 \mathrm{~mA}$. Anesth Analg. 2007; 105:848-852.

17. Amanda A, Boyko E, Ahroni J, Stensel V, Forsberg R, Smith D. Risk factors for diabetic peripheral sensory neuropathy: results of the Seattle prospective diabetic foot study. Diabetes Care. 1997;20:1162-1167.

18. Partanen J, Niskanen L, Lehtinen J, Mervaala E, Siitonen O, Uusitupa M. Natural history of peripheral neuropathy in patients with non-insulin dependent diabetes mellitus. N Engl J Med. 1995;333:89-94.

19. Sites BD, Gallagher J, Sparks M. Ultrasound guided popliteal block demonstrates an atypical motor response to nerve stimulation in 2 patients with diabetes. Reg Anesth Pain Med. 2003;28:479-482.

20. Rigaud M, Filip P, Lirk P, Fuchs A, Gemes G, Hogan Q. Guidance of block needle insertion by electrical nerve stimulation: A pilot study of the resulting distribution of injected solution in dogs. Anesthesiology. 2008;109:473-478.

21. Marhofer P, Schrogendorfer K, Koinig H, et al. Ultrasonographic guidance improves sensory block and onset time of three-in-one blocks. Anesth Analg. 1997;85:854-857. 1997.

22. Marhofer P, Schrogendorfer K, Wallner T, et al. Ultrasound guidance reduces the amount of local anesthetic for 3-in-1 blocks. Reg Anesth Pain Med. 1998;23:584-588.

23. Capdevila X, Biboulet P, Morau D, Bernard N, Deschodt J, Lopez S, d'Athis F. Continuous three-in-one block for postoperative pain after lower limb orthopedic surgery: where do the catheters go? Anesth Analg. 2002;94:1001-1006.

24. Helayel PE, da Conceição DB, Pavei P, Knaesel JA, de Oliveira Filho GR. Ultrasound guided obturator nerve block: A preliminary report of a case series. Reg Anesth Pain Med. 2007;32:221-226.

25. Soong J, Schafhalter-Zoppoth I, Gray AT. Sonographic imaging of the obturator nerve for regional block. Reg Anesth Pain Med. 2007;32:146-151.
26. Ng I, Vaghadia H, Choi PT, Helmy N. Ultrasound imaging accurately identifies the lateral femoral cutaneous nerve. Anesth Analg. 2008;107:1070-1074.

27. Hurdle MF, Weingarten TN, Crisostomo RA, Psimos C, Smith J. Ultrasound guided blockade of the lateral femoral cutaneous nerve: technical description and review of 10 cases. Arch Phys Med Rehabil. 2007;88:1362-1364.

28. Chan VW, Nova H, Abbas S, McCartney CJ, Perlas A, Xu DQ. Ultrasound examination and localization of the sciatic nerve: a volunteer study. Anesthesiology. 2006;104:309-314.

29. Domingo-TriadoV, SelfaS, Martinez F, et al. Ultrasound guidance for lateral mid-femoral sciatic nerve block: a prospective, comparative, randomized study. Anesth Analg. 2007;104:1270-1274.

30. Sites BD, Spence BC, Gallagher JD, Wiley CW, Bertrand ML, Blike GT. Characterizing novice behavior associated with learning ultrasound guided peripheral regional anesthesia. Reg Anesth Pain Med. 2007;32:107-115.

31. Sinha A, Chan VW. Ultrasound imaging for popliteal sciatic nerve block. Reg Anesth Pain Med. 2004;29:130-134.

32. Delgado-Martínez AD, Marchal JM, Molina M, Palma A. Forefoot surgery with ankle tourniquet: complete or selective ankle block? Reg Anesth Pain Med. 2001;26:184-186.

33. Wassef MR. Posterior tibial nerve block. A new approach using the bony landmarks of the sustentaculum tali. Anesthesia. 1991;46:841-844.

34. Palmisani S, Arcioni R, Di Bendetto P, De Blasi RA, Mercieri M, Ronconi P. Ropivacaine and levobupivacaine for bilateral selective ankle block in patients undergoing hallux valgus repair. Acta Anesthesiol Scand. 2008;52:841-844.

35. Redborg KE, Sites BD, Gallagher JD, Ball PA, Antonakakis JG, Beach ML. Ultrasound improves the success rate of a sural nerve block at the ankle. Reg Anesth Pain Med. 2008. In Press.

36. Redborg KE, Antonakakis JG, Beach ML, Chinn CD, Sites BD. Ultrasound improves the success rate of a tibial nerve block at the ankle. Reg Anesth Pain Med. 2008. In Press. 